\title{
Addendum
}

\section{Novel and Convenient Aldolisation of Methyl 3,3,3-Trifluoropyruvate Using Enamines Instead of Ketones}

Jiří Palećek, Oldřich Paleta* Synthesis 2004, 521.

The paper should include references to the recent papers ${ }^{1-3}$ that describe analogous reactions of 1,1,1-trifluoropropan2-one and trifluoroacetaldehyde hydrate. ${ }^{1-3}$

The authors apologize for omitting these references.

\section{References}

(1) Funabiki, K.; Nojiri, M.; Matsui, M.; Shibata, K. Chem. Commun. 1998, 2051.

(2) Funabiki, K.; Isomura, A.; Yamaguchi, Y.; Hashimoto, W.; Matsunaga, K.; Shibata, K.; Matsui, M. J. Chem. Soc., Perkin Trans. 1 2001, 2578.

(3) Funabiki, K.; Matsunaga, K.; Nojiri, M.; Hashimoto, W.; Yamamoto, H.; Shibata, K.; Matsui, M. J. Org. Chem. $2003,68,2853$. 